\title{
Semantic memory for metaphor: The conceptual base hypothesis
}

\author{
RICHARD P. HONECK, PAUL RIECHMANN, and ROBERT R. HOFFMAN \\ University of Cincinnati, Cincinnati, Ohio 45221
}

\begin{abstract}
The idea that people can encode and use an extremely abstract and general form of a complex linguistic (proverb) input - a conceptual base-was examined in two experiments. In Experiment I, each proverb was accompanied by either a conceptually related (good, mediocre, or poor) or an unrelated interpretation. The related interpretations were more effective recall prompts than were the unrelated interpretations, but only for high-imagery proverbs. In Experiment II, subjects wrote interpretations of the proverbs and then received either the proverb subject-noun or a brief story as a prompt. As was the case for the interpretations in Experiment I, the stories did not share any major vocabulary or propositional structure with their proverb source. Nonetheless, the stories were as effective as the nouns. Also, quality of proverb interpretation and of recall performance were positively related, with the correlations involving low-imagery proverbs, and stories, tending to be higher. Both experiments provided support for the conceptual-base notion, and underlined the importance of interpretive context, but more definitive evidence is needed.
\end{abstract}

Extending recent studies (Bransford, Barclay, \& Franks, 1972; Honeck, 1971; Kintsch \& Monk, 1972) on semantic memory, Honeck (1973a) found better memory for a proverb if it was accompanied during acquisition by a conceptually good interpretation than by a repetition of its deep structure. This result leads to two conclusions. First, since the interpretations did not overlap their proverb bases on a deep structure or "propositional structure" basis, subjects apparently can encode an abstract, possibly imagery free, nonlinguistic form of a linguistic input-a conceptual base. Second, the result supports Bransford and Johnson's (1973) findings that long-term understanding can be highly dependent on the presence of appropriate context during initial processing.

Two experiments are reported below which further examine the conceptual base hypothesis and the effects of interpretive linguistic context on semantic memory. Both experiments used proverbs because they can be processed on different semantic levels (cf. Honeck, 1973a, for a complete rationale), which can, in turn, effect different strengths and forms of memory (Craik \& Lockhart, 1972). In Experiment $I$, the acquisition relationship between the to-be-recalled proverbs and interpretive context was experimenter controlled, whereas in Experiment II it was subject controlled. Specifically, in Experiment $I$ the interpretations were also used as recall prompts, with the expectation that interpretations closer to the intended meaning of a proverb would be better prompts. In Experiment II, the subjects wrote their own interpretations of the proverbs during acquisition. Proverb recall was then prompted by either the logical subject-noun of the proverb or by a

This research was supported, in part, by a grant from Albert Steiner to the University of Cincinnati, and by a Summer Faculty Fellowship from the University of Cincinnati to the first author. short "story" which illustrated the proverb. Like the interpretations in Experiment $I$, the stories were constructed such that they retained a conceptual link with the proverbs without relying on propositional structure overlap to do so. Thus, a literal encoding of the proverb, whether in terms of imagery or propositional structure, would preclude subjects using the story prompts to retrieve the correct proverb. By implication, if the stories are effective retrieval agents, then subjects must have encoded something more conceptual than literal imagistic or propositional forms.

Perhaps a working distinction between "propositional structure" and "conceptual base," should be made. As used in Honeck (1973b), propositional structure refers to the logical relationships between the morphemes of a linguistic construction. For example, in the proverb, Great weights hang on small wires, we know that there is a relationship of hanging, that weights hang on wires, that the weights are great, and the wires small. By contrast, a conceptual base is considered to be a recoding of propositional structure into a new form. In our example, this form could be expressed as the interpretation, The outcome of important events often depends on seemingly minor details. Thus, propositional structure constrains the content of the conceptual base, but is distantly related to it because knowledge of the world also contributes to the base.

\section{EXPERIMENT I}

\section{Method}

Subjects. Thirty-two students from introductory psychology courses at the University of Cincinnati received course credit for their participation.

Materials. Twenty proverbs were used. Most were selected from Smith and Heseltine (1935) and the remainder from Palmeri (1963) and Christy (1898). Only those proverbs were 
Table 1

Sample of Proverbs and Interpretations*

There is a great force hidden in a sweet command.

Good A little kindness often persuades better than a rough order.

Mediocre Friends are better able to persuade us than strangers.

Poor A good leader can persuade without abusing power.

Unrelated Increasingly more people are being persuaded to live in the city.

Reputation is commonly measured by the acre.

Good Someone's character may be judged more on the basis of his material goods than his moral fiber.

Mediocre Someone's character often stands on what his associates say about him.

Poor Someone's character is either good or bad depending on the choices he has made.

Unrelated Someone's character is laid down in infancy, according to certain authorities.

*The proverbs are in italics. The upper proverb is high imagery and the lower is low imagery.

selected which, in the authors' opinions, were unfamiliar to college students without exceeding their vocabulary, did not require special knowledge for their interpretation, conformed to more familiar, nonarchaic English phrase structure patterns, contained no rhyme, and repeated no content words within or between proverbs. Four proverbs were buffers and 16 were experimental sentences. The latter varied in length from 6 to 15 words and, in content words, from 4 to 8 . Moreover, the 16 experimental sentences had been rated on a 9-point imagery scale by an independent group of 20 subjects. Both low-imagery $(\overline{\mathrm{X}}=4.76 ; \mathrm{SD}=.66)$ and high-imagery $(\overline{\mathrm{X}}=7.16 ; \mathrm{SD}=.59)$ proverbs were derived from these ratings. Each imagery level contained 48 content words of which 29 were from the AA, 8 from the $A$, and 11 from the $1-49$ per million Thorndike-Lorge frequency categories. Also, each imagery level possessed 25 propositions.

A good, mediocre, poor, and unrelated interpretation was also constructed for each experimental proverb. Unrelated interpretations were constructed which did not logically confirm or contradict their proverb base. The good, mediocre, and poor interpretations were constructed on the basis of the authors' independently made ratings, on a 7-point scale, of 10 different subjects' interpretations of the proverbs. Only those interpretations were selected which met a two-thirds agreement criterion on a particular category, good being defined as 1 or 2 on the rating scale, mediocre as 3 or 4 , and poor as 5,6 , or 7 . Interjudge agreement was quite good, as, overall, only $6 \%$ of the interpretations produced a three-way split. The interpretations used were tailored so that they did not share any content words with their corresponding proverb, used the same synonym for a given proverb content word if that synonym was used for any given interpretation, possessed no unduly complex vocabulary or phrase structure, and were, overall, approximately equal in length. Furthermore, content word overlap between the 20 interpretations heard by a given subject was minimized in order to prevent any confusion between the interpretations and/or proverbs during recall. Also, none of the interpretations shared the major vocabulary, phrase structure, deep structure, or basic propositional structure of their corresponding base proverb. A sample of the proverbs and corresponding interpretations is provided in Table 1 .

Design and procedure. The experiment had two stages. In Stage 1 , all subjects were given 20 trials, a trial being defined by the presentation of four sentences. More specifically, a trial proceeded as follows: Sentence 1 (the proverb); 2-sec pause; Sentence 2 (the interpretation); $2-\sec$ pause; Sentence 3 , a repetition of the proverb; 2 -sec pause; Sentence 4, a repetition of the interpretation; 2 -sec pause; the instruction "next," indicating that a new set of four sentences would be presented in $2 \mathrm{sec}$. Two buffer trials were inserted at the beginning and two at the end of the series with the 16 experimental trials sandwiched between. These latter trials consisted of four four-trial blocks such that each interpretation level occurred in each block. A Latin-square technique was used to counterbalance any order effects and to balance interpretation level across proverbs. Eight subjects were tested on each of the four orders of the square. Proverb imagery level was counterbalanced within blocks such that each level occurred twice within a block and twice at each interpretation level across all blocks. Thus, a completely within-subjects design, in which interpretation level was completely crossed with imagery level, was used.

The subjects were tested chiefly as groups of size four to eight, although a few were tested individually. All subjects were instructed that the sentences would come in sets of four, to try to relate the proverb and interpretation in each set, and to remember as much as they could. The instructions and the four different orders were recorded with normal intonation on a Wollensak tape recorder. Stage 1 lasted approximately $15 \mathrm{~min}$.

Two seconds after the last trial of Stage 1, taped instructions were given for Stage 2 recall. The subjects were told to use the interpretations as a basis for written recall of the proverbs associated with them in Stage 1. Instructions requested verbatim recall, if possible, and that an $X$ be placed on a booklet page if nothing could be recalled. Each tape-recorded interpretation was presented twice, with a $2-\mathrm{sec}$ intersentence interval, and followed by a 45 -sec recall interval. The word "turn" was then presented, cuing subjects to turn the booklet page and prepare to listen for the next interpretation. Interpretations of the four buffer proverbs were presented first, in order to further insure that, along with instructions and booklet distribution, retrieval from a short-term acoustic or phonetic store would be minimized. The buffers were not scored for recall. The following 16 experimental interpretations were presented in the same order as they occurred in Stage 1 . Stage 2 lasted approximately $20 \mathrm{~min}$.

\section{Results}

Three measures of recall performance were taken, content words recalled in any order (WA), content words recalled in the correct order (WC), and propositions recalled (PR). The mean performance on these measures for each condition is given separately in Table 2 for low- and high-imagery proverbs.

An analysis of variance on content words recalled in any order (WA) revealed a significant interpretations effect, $F(3,93)=5.98, p<.001$. A Duncan range test on this main effect indicated, at the .05 level, that good, mediocre, and poor interpretations were equally effective but only the goods were more effective than the unrelateds. Also, more content words were recalled from high-imagery proverbs than from low-imagery proverbs, $F(1,31)=20.46, p<.001$. Finally, the Interpretations by Imagery Level interaction was not significant, at the .05 level, although there was a trend, $F(3,93)=2.37, p<.10$.

An analysis of variance on content words recalled in the correct order (WC) revealed a significant interpretations effect, $F(3,93)=5.79, p<.005$. As in the WA analysis, a Duncan range test indicated, at the 
.05 level, that only the good interpretations were more effective than the unrelateds. Second, more content words were recalled from high-imagery than from low-imagery proverbs, $F(1,31)=23.09, \quad p<.001$. Finally, the Interpretations by Imagery Level interaction was not significant, although there was a trend, $F(3,93)=2.28, p<.10$.

An analysis of variance was also computed on propositions (PR) recalled. A proposition was scored correct if, in the authors' opinion, it was a paraphrase of, or, more generally, it retained the semantic structure of the corresponding proverb proposition. Thus, whether subjects used the same or different, but synonymous, constituents (usually content words) made no difference in scoring. Note that certain precautions were taken in scoring propositions so that the scores for the better interpretations were not spuriously inflated. Such inflation could have occurred either because the subjects simply repeated part or all of the interpretation, transformed the interpretation without changing its major vocabulary, or wrote a paraphrase of the interpretation during recall. Obviously, if such strategies were allowed, scores for the better interpretations would be higher simply because their semantic structure was closer to their corresponding proverbs. Therefore, the following criteria were used to decide that subjects' protocols evidenced a reasonable attempt to recall the proverb: (a) no content word from the interpretation could be present; (b) at least one content word from the proverb had to be used, or, if no such content word was used, (c) the material recalled could not, in the authors' opinion, constitute a paraphrase of the interpretation. Actually, scoring of propositions was relatively easy because $90 \%-95 \%$ of the propositions recalled contained at least one content word from the appropriate proverb.

The analysis of propositions recalled indicated a highly significant effect due to interpretations, $F(3,93)=14.61, p<.001$. Further, more propositions were recalled from high-imagery proverbs than from low-imagery proverbs, $F(1,31)=26.99, \mathrm{p}<.001$.

Table 2

Mean Recall Performance*

\begin{tabular}{|c|c|c|c|c|c|c|c|c|}
\hline \multirow{3}{*}{$\begin{array}{l}\text { Mea- } \\
\text { sure }\end{array}$} & \multirow{2}{*}{\multicolumn{2}{|c|}{ Good }} & \multirow{2}{*}{\multicolumn{4}{|c|}{$\begin{array}{cc}\text { Interpretations } \\
\text { Mediocre }\end{array}$}} & \multirow{2}{*}{\multicolumn{2}{|c|}{ Unrelated }} \\
\hline & & & & & & & & \\
\hline & Low & High & Low & High & Low & High & Low & High \\
\hline WA & $\begin{array}{l}3.34 \\
2.44\end{array}$ & $\begin{array}{l}4.75 \\
2.46\end{array}$ & $\begin{array}{l}2.47 \\
2.50\end{array}$ & $\begin{array}{l}4.41 \\
2.55\end{array}$ & $\begin{array}{l}2.72 \\
2.73\end{array}$ & $\begin{array}{l}3.91 \\
2.79\end{array}$ & $\begin{array}{l}2.34 \\
2.74\end{array}$ & $\begin{array}{l}2.31 \\
2.62\end{array}$ \\
\hline WC & $\begin{array}{l}3.16 \\
2.29\end{array}$ & $\begin{array}{l}4.47 \\
2.48\end{array}$ & $\begin{array}{l}2.38 \\
2.43\end{array}$ & $\begin{array}{l}4.12 \\
2.45\end{array}$ & $\begin{array}{l}2.19 \\
2.13\end{array}$ & $\begin{array}{l}3.62 \\
2.61\end{array}$ & $\begin{array}{l}2.22 \\
2.62\end{array}$ & $\begin{array}{l}2.25 \\
2.58\end{array}$ \\
\hline PR & $\begin{array}{l}2.78 \\
2.14 \\
\end{array}$ & $\begin{array}{l}3.84 \\
1.67 \\
\end{array}$ & $\begin{array}{l}1.34 \\
1.60\end{array}$ & $\begin{array}{l}3.15 \\
2.08\end{array}$ & $\begin{array}{l}1.75 \\
1.55\end{array}$ & $\begin{array}{l}3.16 \\
2.03 \\
\end{array}$ & $\begin{array}{l}1.37 \\
1.70 \\
\end{array}$ & $\begin{array}{l}1.34 \\
1.58\end{array}$ \\
\hline
\end{tabular}

* Low and high refer to imagery levels. The scores in each cell represent the mean (above) and SD (below) of two trials. The maximum mean is 6 for WA (content words in any order), 6 for WC (content words in correct order), and 6.25 for $P R$ (propositions).
Table 3

Sample Proverbs and Story Prompts

Story Two people had their day in court. One was found guilty of smoking marijuana, was fined $\$ 500$ and sent to jail for 6 months. The second person was charged with not reporting $\$ 100,000$ in income. He was simply reprimanded by the judge and told to pay the government what he owed. In comparing these two cases, a courtroom spectator said-

Proverb Laws catch flies but let hornets go free.

Story A jewel thief was successful for a long time. However, a detective caught him after 10 years. But the detective said that if he had not caught the thief someone else would have because-

Proverb In due time, the fox is brought to the furrier.

Finally, the Interpretations by Imagery Level interaction was significant, $F(3,93)=3.80, p<.025$. A Duncan range exploration of this interaction, whose pattern was the same for the WA and WC analyses, indicated that only the unrelated interpretations of high-imagery proverbs failed to surpass recall from the corresponding low-imagery proverbs. Furthermore, the good, mediocre, and poor interpretations of high-imagery proverbs were equal, but superior to the unrelated interpretations of high-imagery proverbs. However, good interpretations of low-imagery proverbs were more effective than mediocre, poor, or unrelated interpretations, these latter three conditions being equal.

\section{Discussion}

In general, the results indicate that good, mediocre, and poor interpretations were better prompts for high-imagery proverbs than were unrelated interpretations. The interpretations did not differ in prompt effectiveness for low-imagery proverbs, with the exception that good interpretations were best when propositions were scored. However, because the good, mediocre, and poor interpretations produced basically the same results throughout, we hereafter will refer to them collectively as the related interpretations. In summary, related interpretations were more effective prompts than unrelated interpretations, but only for high-imagery proverbs.

Two explanations of these results are considered. The first appeals to imagery and the second to imagery plus the conceptual-base notion.

Proverb imagery level was a potent factor, as the main effect in the three analyses attests to. Moreover, the Stage 1 procedure is similar to a paired-associates task, and it is well established (Paivio, 1969) that high-imagery stimulus items, proverbs by this analogy, promote more rapid learning than low-imagery items. But why should related interpretations do better as prompts than unrelated interpretations? Perhaps the former gained in imagery value themselves. Alternatively, related context may have imbued the high-imagery proverbs with even greater imagery value. 
In any case, the related interpretations must have possessed special characteristics. This is because no matter what input, proverbs or interpretations, or both, increased in imagery value, and by implication, in availability during recall, it is the proverb-interpretation conceptual relationship that would have to change these input imagery values.

The link between proverb and related interpretation was not logical-propositional or vocabulary based, yet subjects were able to understand something of this relationship. And high-imagery proverbs, because they can be better held in operational memory and/or because they are more interpretable, helped produce the interaction. We conclude, therefore, that subjects remembered conceptual bases, abstract intermediaries between the proverbs and their interpretations, which allowed subjects, when given the interpretation, to "deduce" the correct proverb.

It is not clear why the good, mediocre, and poor interpretations did not produce differential results. Perhaps time pressure and short-term memory load prevented subjects from thinking enough about proverb-interpretation relationships. Alternatively, the interpretations may not have agreed with the subjects' own or with their judgment of relative goodness. Pertinent to this point is evidence from our laboratory indicating that college subjects show strong agreement in their rank ordering of the conceptual similarity of interpretations to proverbs, and that their judgments are practically identical to the authors'.

Finally, the question of whether subjects noticed a conceptual relationship between proverb and interpretation during acquisition, or recall, should be considered. That is, is a conceptual base encoded or retrieved, or both? There are at least two retrieval hypotheses. On the assumption that synonymous sentences allow mutual derivation, subjects could have recalled the correct high-imagery proverb, given a related interpretation, without benefit of any prior exposure to the proverb. This seems extremely improbable. Alternatively, when provided a related interpretation, subjects scanned the set of high-imagery proverbs and selected the most conceptually related. Unfortunately, this view assumes that the proverbs were readily available and rapidly relatable to their proper interpretation. This view is more plausible, but the task emphasized that subjects encode the relationship, if at all possible. Experiment II provides more evidence for the encoding hypothesis by testing subjects generalization capabilities.

\section{EXPERIMENT II}

\section{Method}

Subjects. The subjects were 20 students from introductory psychology courses at the University of Cincinnati who received course credit for their participation.

Materials. Twenty proverbs were used, including 15 from Experiment I. Most were selected, by the same criteria as in Experiment I, from Smith and Heseltine (1935) and the remainder from Palmeri (1963). Four proverbs were used as buffer items and 16 as experimental items. The latter varied in length from 6 to 15 words and, in content words, from 4 to 8 . The experimental sentences were divided into a set of eight low-imagery $(\overline{\mathrm{X}}=4.97 ; \mathrm{SD}=.74)$ and eight high-imagery $(\bar{X}=7.25 ; \mathrm{SD}=.46)$ sentences on the basis of an independent group of 20 subjects' ratings on a 9-point imagery scale. Each imagery level contained 48 content words of which most were from the AA, fewer from the 1-49, and fewer still from the $A$ Thorndike-Lorge frequency categories. The two imagery levels contained approximately equal numbers of words from these categories. Finally, each imagery level contained 24 propositions, 12 each for the two types of recall prompt.

Several short two-to-four-sentence "stories" were also constructed which illustrated a particular proverb. The stories did not retain the major vocabulary or propositional structure of their base proverb, whereas the single subject-noun from each proverb, which was also used as a recall prompt, obviously was part of the major vocabulary of that proverb. See Table 3 for a sample of the proverbs and story prompts.

Design and procedure. The experiment had two stages. In Stage 1, subjects wrote an interpretation for each of the 20 proverbs in a booklet. The first booklet page instructed them to read each proverb for intended meaning and to write an interpretation. The subjects were allowed $40 \mathrm{sec}$ per proverb to read, interpret, and write their interpretation. Twenty-five seconds into this interval a tone sounded, informing subjects that they had 15 sec left to write, although they were at liberty to write at any time during the $40-\mathrm{sec}$ interval. At the end of the interval, the prerecorded word "next" was heard, which signaled subjects to turn the page and begin work on the next proverb. The subjects wrote a proverb out verbatim if they could not interpret it. This rarely happened, however, as is attested to by their protocols. Thus, the $40-\mathrm{sec}$ interval allowed subjects to perform the task comfortably. To reduce primary and recency effects in Stage 2, the first and last two proverbs were buffers, with the 16 experimental proverbs sandwiched between.

After Stage 1, the booklets were collected and new booklets were distributed. The first page instructed subjects that each subsequent page would contain a prompt for verbatim recall of the proverbs and that they could work at their own pace. Recall of the buffers, which were not scored, was solicited first to help prevent retrieval of the experimental items from a short-term acoustic-phonetic store. Recall of the experimental items was prompted by a single subject-noun cue or by a story. For each subject, four of the eight proverbs at each imagery level were prompted by a noun and the other four by a story. Across subjects, however, type of recall prompts was balanced across proverbs so that each proverb was prompted by both types of prompts. Each of the four categories (two imagery levels by two types of prompt) thus formed contained 24 content words. The four categories were block randomized during Stage 1 as well as in Stage 2, except that Block 1 proverbs in Stage 1 were solicted first for recall in Stage 2, Block 2 proverbs in Stage 1 were solicited second for recall, and so on. In sum, the design used was completely within-subjects, with a factorial combination of two levels of proverb imagery and two types of recall prompts.

\section{Results}

Two measures of recall performance were taken, content words recalled in any order (WA), and propositions (PR) recalled. The overall Pearson $r$ between these measures was 94 . The mean performance on these measures is presented in Table 4.

An analysis of variance was computed on the number of content words recalled in any order. Note that the subject-noun cue from each proverb was not scored for this analysis because it was visually present for the noun 
condition, but not in the story prompt, and scoring for its presence in this latter condition would have spuriously inflated performance. The analysis revealed that more words were recalled from high-imagery than from low-imagery proverbs, $\mathrm{F}(1,19)=5.39, \mathrm{p}<.05$, but prompt type, $F(1,19)=.63$, and the Prompt Type by Imagery Level interaction, $F(1,19)=2.40, p>.05$, were not significant.

An analysis of variance was also computed on the number of propositions recalled. A proposition was scored correct if, in the writers' opinion, it retained the semantic structure of the corresponding proverb proposition. Thus, whether subjects used the same or different, but synonymous, constituents (usually content words) made no difference in scoring. The analysis revealed no significant effect due to imagery level, $F(1,19)=1.63, p>.05$, or to prompt type $F(1,19)=.37$. However, the Imagery Level by Prompt Type interaction was significant, $F(1,19)=5.01$, $\mathrm{p}<.05$. A Duncan range exploration of this interaction indicated that stories were more effective prompts for recall of high-imagery than low-imagery proverbs, whereas the noun prompt produced equivalent recall of high- and low-imagery proverbs. Moreover, the story prompts for high-imagery proverbs were marginally better $(\mathrm{p}<.10)$ than noun prompts, but story and noun prompts of low-imagery proverbs did not differ in effectiveness.

An important question concerned the correlation between goodness of proverb interpretation and recall. The authors independently rated the interpretations on a 7-point scale, where 1 meant an excellent interpretation and 7 meant a terrible or no interpretation at all. Interjudge agreement was $95 \%$ on whether an interpretation fell in the good ( 1 and 2 on the scale), mediocre $(3,4$, and 5$)$, or poor (6 and 7$)$ category. For each subject, the mean of the total of the three judges' ratings was then obtained for each of the four proverbs in each of the four experimental conditions. Pearson rs were computed on these means, and the subjects' total recall score for the corresponding four proverbs. Inspection of these correlations in Table 5 indicates that all but one was significantly greater than zero at the .05 level. Thus, the better the interpretation, the better the

Table 4

Mean Recall Performance*

\begin{tabular}{ccccc} 
& \multicolumn{3}{c}{ Prompt Type } \\
Measure & Low & High & Low & High \\
\hline \multirow{2}{*}{ WA } & 8.20 & 8.50 & 7.70 & 10.20 \\
& $(4.19)$ & $(4.72)$ & $(3.20)$ & $(3.78)$ \\
PR & 7.50 & 7.00 & 6.80 & 8.50 \\
& $(3.47)$ & $(3.78)$ & $(2.97)$ & $(2.69)$ \\
\hline
\end{tabular}

"Low and high refer to imagery levels. The maximum mean is 20 for WA (content words in any order) and 12 for PR (propositions). The SDs are in parentheses.
Table 5

Correlation Between Quality of Interpretation and Recall*

\begin{tabular}{ccccc}
\hline & \multicolumn{3}{c}{ Noun } & \multicolumn{2}{c}{ Story } \\
Measure & Low & High & Low & High \\
\hline WA & -.61 & -.39 & -.57 & -.55 \\
PR & -.45 & $-.36^{* *}$ & -.66 & -.52 \\
\hline
\end{tabular}

*Low and high refer to imagery levels. $A$ negative correlation indicates that good interpretations are related to good recall, poor interpretations to poor recall.

**Not significant at the .05 level. All other vahues are significant.

recall. Also, low-imagery proverbs tended to produce higher correlations than high-imagery proverbs and, with one exception, story prompts produced higher correlations for the corresponding measure and condition than did noun prompts.

The foregoing correlational analysis is suspect, however, because one would naturally expect people who interpreted well to recall well, suggesting that the correlations tapped primarily individual differences in verbal intelligence. We should also know what the relationship is for a particular subject. Therefore, for each subject, the total content word-recall scores for each of the four experimental conditions were rank ordered and correlated with the rank order of the interpretations for these conditions. Twenty rank-order correlations, one per subject, were thus computed. The median rho, based on four paired ranks per rho was .40 , corroborating the prior conclusion that goodness of interpretation and recall are directly, though not strongly, related.

\section{Discussion}

The pattern of evidence presents a good case for the conceptual base construct. The basic premises are that the stories were conceptually related particularizations of the proverb metaphors, and that the interpretations sharing a conceptual link with the proverbs were general or coordinate rather than particular characterizations and were not specifically related to the stories.

The subjects recognized the stories as particularizations of the conceptual meaning of the proverbs, at least as well as they could recall the proverbs when given the noun prompt. However, since the subjects' interpretations were, in a sense, intermediary between the proverbs and the stories, they must also be considered. First, interpretation quality was inversely related to the extent of major vocabulary or propositional structure overlap between a proverb and an interpretation. This occurred because "interpretations" which simply repeated the proverb, transformed its word order, or substituted synonyms, were judged as poor interpretations. Similarly, for proverbs actually interpreted, that is, for proverbs not simply repeated, left blank, or whose interpretations were incomplete, the major vocabulary overlap was 
approximately $20 \%$. Specifically, the mean proverb-interpretation content word overlap was $18.1 \%$ for high-imagery proverbs and $22.7 \%$ for low-imagery proverbs. Second, these interpretations were almost inevitably general, rather than particular characterizations of the proverbs. And in no case did a subject write anything which resembled the story prompt for the proverb. Third, the relationships between interpretation quality and recall were positive, with those involving the stories tending to be highest. Fourth, low-imagery proverbs tended to produce higher correlations than high-imagery proverbs, suggesting, in conjunction with the preceding points, that the better the general context subjects could provide for abstract inputs, the better they could recall them. We conclude from this extended argument that people can encode an abstract characterization of a linguistic input, a conceptual base, which they can then use as a mediating device to recognize specific, conceptually related, instances thereof. Moreover, the correlational data, in particular, suggest that appropriate context may be necessarv for the formation of this base.

The results also bear on the encoding specificity principle which asserts that "only that can be retrieved that has been stored, and that how it can be retrieved depends on how it was stored" (Tulving \& Thompson, 1973 , p. 359). Although we agree with these authors' assertion that recall is closely tied to subjects' operations on the input, we emphasize that insofar as a conceptual base is remembered, then even so remote a prompt as similar or implied instruments, agents, objects, etc., as defined by case grammar (Fillmore, 1968), do not do justice to people's generalization capabilities. For example, what are the case relationships between the proverb, In due time the fox is brought to the furrier, and subjects' actual interpretations such as, Your actions are bound to be accounted for eventually, Everything comes home, and Any problem will be solved eventually? In general, no extant theory adequately describes the semantic relationships between the proverbs, subjects' interpretations, and corresponding story. Moreover, this example points up the circularity of the encoding specificity principle. Since we do not know what people remember until we test their memory, we are only able to describe what they remember by hindsight. Hence, even if people remember a highly abstracted form of the input, the theory still "predicts" the effectiveness of a prompt because it is almost always possible to discover a similarity between two events. Obviously, we should have to specify the nature of what is remembered and what is similarity, especially semantic similarity, more formally.

\section{General Discussion}

The results of both experiments reinforce several ideas about semantic memory. Bransford and Johnson (1973) emphasize that such memory is exceedingly dependent on the presence of relevant context during acquisition. This was demonstrated directly by Experiment I and is implicit, in Experiment II, in the moderate correlations between interpretation quality and recall and particularly in the enhanced correlations involving low-imagery proverbs. In general, these findings dovetail with Bobrow and Bell's (1973) that distinct literal vs. metaphorical processing sets or modes can arise.

Both experiments also point to the inadequacy of the "interpretive" view of semantic memory (Bransford et al., 1972), since subjects were apparently using more than deep structural information. Verbrugge and McCarrell (Reference Note 1), who used sentence idioms, also found that unexpressed acquisition information can function as an effective prompt. This finding, Bobrow and Bell's (1973), Honeck's (1973a), and our own vindicate the use of metaphorical materials in exploring semantic phenomena. In particular, we agree with Verbrugge and McCarrell's suggestion that processing of metaphorical material may not be different from that encountered under natural conditions of language understanding.

In both experiments, proverb imagery level interacted with another factor, whereas in Honeck (1973a) no such interaction occurred. All three experiments used a prompted free-recall paradigm, but the prompts and/or the details of the acquisition procedure were different. A noun prompt was used in Honeck (1973a) and in Experiment II wherein the noun prompt, but not the story prompt, was not differentially effective for recall of low-vs. high-imagery proverbs. The proverbs also differed slightly from experiment to experiment, a factor which is made more salient because recent data from our laboratory indicates that it is possible to generate proverb sets which are orthogonal on imagery (low vs. high) and comprehensibility (easy vs. hard) dimensions. In short, the reasons for the discrepancy are unclear.

Finally, the conceptual base notion is not a unique one. Similar ideas have been proposed by Chase and Clark (1972), Kintsch (1972), Pylyshyn (1973), Shank (1972), Simon (1972), and Winograd (1972). Unfortunately, the conceptual base construct has not been formalized, but it does have some interesting and testable consequences. For example, if this base is abstract and imagery free, then subjects instructed to encode proverbs for intended meaning should better recognize interpretations of those proverbs than subjects instructed to image the literal meaning of the proverbs, a result just obtained by Riechmann (1974) in our laboratory.

\section{REFERENCE NOTE}

1. Verbrugge, R. R., \& McCarrell, N. S. The role of inference in the comprehension of metaphor. Paper presented 
at the meeting of the Midwestern Psychological Association, Chicago, May 12, 1973.

\section{REFERENCES}

Bobrow, S. A., \& Bell, S. M. On catching on to idiomatic expressions. Memory \& Cognition, 1973, 1, 343-346.

Bransford, J. D., Barclay, J. R., \& Franks, J. J. Sentence memory: A constructive versus interpretive approach Cognitive Psychology, 1972, 3, 193-209.

Bransford, J. D., \& Johnson, M. K. Considerations of some problems of comprehension. In W. G. Chase (Ed.), Visua information processing. New York: Academic Press, 1973. Pp. 383438.

Chase, W. G., \& Clark, H. H. Mental operations in the comparison of sentences and pictures. In L. W. Gregg (Ed.), Cognition in learning and memory. New York: Wiley, 1972. Pp. 205-252.

Christy, R. Proverbs, maxims, and phraves of all ages. New York: Putnam, 1898.

Craik, F. I. M., \& Lockhart, R. S. Levels of processing: A framew ork for memory research. Journal of Verbal Learning and Verbal Behavior, 1972, 11, 671-684.

Fillmore, C. J. The case for case. In $\mathrm{E}$. Bach and R. T. Harms (Eds.), Universals in linguistic theory. New York: Holt, Rinehart, and Winston, 1968.

Honeck, R. P. A study of paraphrases. Journal of Verbal Learning and Verbal Behavior, 1971, 10, 367-381.

Honeck, R. P. Interpretive versus structural effects on semantic memory. Journal of Verbal Learning and Verbal Behavior, $1973 a, 12,448-455$

Honeck, R. P. Semantic similarity between sentences. Journal of Psycholinguistic Research, 1973b, 2, 137-151.
Kintsch, W. Notes on the semantic structure of memory. In E. Tulving and W. Donaldson (Eds.), Organization of memory. New York: Academic Press, 1972.

Kintsch, W., \& Monk, D. Storage of complex information in memory: Some implications of the speed with which inferences can be made. Journal of Experimental Psychology, 1972, 94, 25-32.

Paivio, A. Mental imagery in associative learning and memory. Psychological Review, 1969, 76, 241-263.

Palmeri, J. French uit and wisdom. Madison: Strauss, 1963.

Pylyshyn, W. W. What the mind's eye tells the mind's brain: A critique of mental imagery. Psychological Bulletin, 1973, 80, $1-24$.

Riechmann, P. F. Does imagery facilitate memory for conceptual information? Unpublished doctoral dissertation. University of Cincinnati, 1974.

Shank, R. C. Conceptual dependency: A theory of natural language understanding. Cognitive Psychology, 1972, 3, $552-631$.

Simon, H. A. What is visual imagery? An information processing interpretation. In L. W. Gregg (Ed.), Cognition in learning and memory. New York: Wiley, 1972.

Smith, W. G., \& Heseltine, J. E. The Oxford dictionary of English proverbs. Oxford: Clarendon Press, 1935.

Tulving, E., \& Thompson, D. M. Encoding specificity and retrieval processes in episodic memory. Psychological Review, $1973,80,352-373$.

Winograd, T. Understanding natural language. Cognitive Psychology, 1972, 3, 1-101.

(Received for publication July 23, 1974; revision received October $4,1974$. ) 